\title{
The 2018 Nobel Prize in Chemistry: phage display of peptides and antibodies
}

\author{
Rodrigo Barderas $^{1}$ (D) Elena Benito-Peña ${ }^{2}$
}

Received: 30 January 2019 / Accepted: 25 February 2019 / Published online: 19 March 2019

(C) Springer-Verlag GmbH Germany, part of Springer Nature 2019

\begin{abstract}
One-half of the 2018 Nobel Prize in Chemistry was awarded jointly to George P. Smith and Sir Gregory P. Winter "for the phage display of peptides and antibodies". This feature article summarizes significant achievements leading to the development of phage display of peptides and antibodies, where a bacteriophage is genetically modified to display peptides and proteins, with the primary aim of producing new biopharmaceuticals. These significant achievements are proven to be useful for the development of phage-based bioassays and biosensors.
\end{abstract}

Keywords Nobel Prize 2018 · Phage display of peptides and proteins · Biopharmaceuticals · Therapeutic antibodies

\section{Introduction}

Bacteriophages or phages are ubiquitous viruses in nature, but harmless to humans. Their first biotechnological application, taking advantage on the fact that some phages can destroy bacterial cells, was as therapeutic tools against some bacterial infections in the early 1900s [1, 2]. In the 1940s, the development of antibiotics ended this specific use $[1,2]$. However, since the invention of phage display of peptides and proteins in the late 1980s and 1990s, the initial use of bacteriophages was boosted from clinical applications, such as identification of ligands, evaluation of protein-protein interactions, or production of antibodies, to the multiple implementations in which they are used nowadays, including the construction of nanotubes, nanobatteries, or nanorods $[3,4]$ and, in recent years, for analytical biosensing $[5,6]$. Notably, phages rarely require inoculation and killing of animals [7]; thus, the production of phages for multiple applications is simple and economical.

Rodrigo Barderas

r.barderasm@ isciii.es

Elena Benito-Peña

elenabp@quim.ucm.es

1 Functional Proteomics Unit, UFIEC, Chronic Disease Programme, Instituto de Salud Carlos III, Majadahonda, 28220 Madrid, Spain

2 Departamento de Química Analítica, Facultad de Ciencias Químicas, Universidad Complutense de Madrid, 28040 Madrid, Spain
Phages are extraordinarily robust and stable virus particles, which use bacterial cells as hosts for replication. Phages are composed of a protein coat that encapsulates its RNA or DNA genome, comprising from four to hundreds of genes [8]. The advantage of phages is that coat proteins can be readily conjugated or genetically engineered to display peptides, proteins, or antibodies [7, 8]. Escherichia coli phages-M13 filamentous phages and their close relatives fd and $\mathrm{fl}$-are the most extensively used, owing to their ease of culture and quick amplification, which makes their production fast and economical. All coat proteins of M13 phages have been utilized to display either foreign peptides or small to large proteins; however, the minor coat protein pIII is the most widely used, followed by the major coat protein pVIII $[8,9]$. Upon construction of phage-libraries, which consist of billions of different displayed peptides or protein variants, subsequent screening allows the identification of specific peptides or antibodies against the desired target molecule, yielding a variety of inexpensive, accurate, and efficient applications in multiple research fields.

Since the development of phage display in the 1980 s, its use has exponentially expanded. Indeed, phage display has become an invaluable tool in so many applications (Fig. 1) $[6,8,10]$. This technology has permitted the production of the first six commercialized antibody medicines, with many more based on human antibodies and antibody fragments in the pipeline. In recognition of these advances, because they have revolutionized chemistry and the development of biopharmaceuticals, one-half of the 2018 Nobel Prize in 


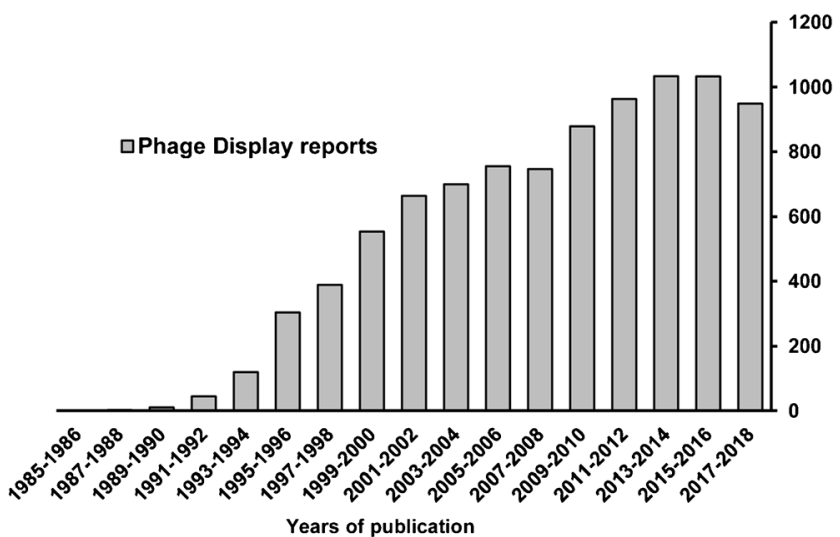

Fig. 1 Number of reports using phage display. Note that the number of reports has increased exponentially from the initial manuscript describing the technology. Its multiple applications have led to a consistent number of manuscripts using the technology in recent years

Chemistry was awarded to George P. Smith and Sir Gregory P. Winter "for the phage display of peptides and antibodies".

\section{The development of phage display}

Phage display represents a leading technological breakthrough developed by George P. Smith. Because it is possible to link a protein with the genetic machinery that encodes it, phage display is a practical realization of artificial chemical evolution [8], and thus, confers on proteins the key properties of replicability and mutability. Using standard recombinant DNA technology, peptides (or proteins) are associated with replicating viral DNAs that include the coding sequences of peptides. The peptide populations created are managed by simple microbiological methods.

The foreign DNA codifying for a specific protein member of a library is packaged inside the genome of the phage in such a manner that the foreign sequence is fused to the endogenous amino acid sequence of a coat protein of the phage to make a hybrid fusion protein. Subsequently, the hybrid coat protein is incorporated into the phage and released from the host cell, so that the foreign peptide or protein is displayed on its surface. This technology simplifies the screening, identification, and amplification of phages displaying the best binding proteins for the query under investigation. The subsequent infection and amplification of phages displaying the best binders in $E$. coli enable the enrichment of specific phages in the library.

The original paper describing the technology was published in 1985 [11]. Therein, Smith reported that the DNA codifying for a 57 -mer peptide could be inserted into the middle of the filamentous phage gene III to create a fusion protein with the foreign peptide sequence in a loop of coat protein III.
The fusion protein was displayed "in an immunologically accessible form" on the surface of the phage, which retained its infectivity capability. Through a single round of enrichment by affinity purification using an antibody against the foreign sequence, Smith showed that those phages displaying the foreign sequence were enriched more than 1500 -fold over other phages. From these findings, Smith suggested that it would be possible to isolate desired clones from a library of random inserts in a fusion-phage vector [11]. As expected, this prediction was proven in subsequent reports that were seminal contributions to the full development of phage display of peptide libraries $[12,13]$.

\section{Phage display of peptides}

In 1988, Smith and his PhD student, Stephen F. Parmley, achieved remarkable improvements to the phage display technology [12]. For example, they moved the cloning site of foreign DNA sequences into the pIII protein to obtain a fully functional pIII protein. This modification was essential for the propagation of phages through the affinity purification of target phages from a library of a million or billions of clones (termed biopanning), which considerably reduced the antibody requirements compared with the original procedure published in 1985 [12].

Next, in the 1990s, the identification of antibody epitopes was accomplished through the construction of combinatorial filamentous phage libraries containing 40 million 6-mer peptides [13], or 20 million 15-mer peptides [14]. In these manuscripts describing epitope mapping of antibodies, phage display peptide libraries were constructed using the framework for producing the libraries fused to the pIII protein developed by Smith for the selection of binding peptides. After three rounds of biopanning, the highest affinity peptides measured by ELISA were retained, and a consensus binding motif for the antibody epitopes was deduced after DNA sequencing of monoclonal phages.

The high value of these reports, as predicted by Smith, consisted of the effective survey for specific affinity epitopes of an antibody, receptor, or other binding proteins by simple recombinant DNA methods. What Smith probably could not imagine was the vast number of applications of his invention in so many fields, with high commercial impact within a very short period of time.

As its initial application, the phage display of peptide libraries made it possible to determine the epitopes of antibodies. The opposite situation, when the molecule displayed on phages is an antibody, was made possible owing to a breakthrough step towards the production of in vitro antibodies against any target or the development of therapeutic antibodies. 


\section{Phage display of antibodies}

The antibody molecule is a sizeable bivalent molecule of $150 \mathrm{kDa}$ composed of a heavy and a light chain. Due to this size, the display of this molecule on the surface of a phage is very challenging. Therefore, smaller, monovalent antibody fragments that retain affinity properties, such as single-chain variable fragments $(\mathrm{scFvs})(\approx 28 \mathrm{kDa})$ and antigen-binding fragments (Fabs) $(\approx 50 \mathrm{kDa})$, have been chosen for the successful display of antibodies on phages. scFvs are recombinant antibody fragments with variable regions of light $\left(\mathrm{V}_{\mathrm{L}}\right)$, and heavy $\left(\mathrm{V}_{\mathrm{H}}\right)$ immunoglobulin chains joined by a flexible polypeptide linker. scFvs are smaller than Fabs which are composed of the whole light chain, $\mathrm{C}_{\mathrm{H}} 1$, and the variable region of the heavy chain of an antibody. Both $\mathrm{scFv}$ and $\mathrm{Fab}$ antibody formats have been successfully used in antibody libraries displayed on phages $[9,15,16]$. However, initial efforts were focused on the display on phages of scFvs encoding antigen-binding domains engineered into a single polypeptide [17].

Several groups, namely, the Smith, Winter, and Lerner groups, pursued the display of antibody fragments on phages, as well as the construction of combinatorial antibody phage display libraries. However, the group of Sir Gregory P. Winter was able to present the first reports demonstrating the successful display of the $\mathrm{scFv}$ of an antibody on the phage used by Smith (fd-tet phage), the construction of combinatorial scFv phage display antibody libraries, and the possibility to humanize murine antibodies using human antibody scaffolds [17-19].

Winter and co-workers, in an original work on displaying an antibody fragment on a phage, showed three remarkable achievements: (1) fd bacteriophages could display scFv antibody fragments on their surface; (2) phages displaying the $\mathrm{scFv}$ bound to its specific target, and (3) one in a million specific engineered phages could be isolated by affinity chromatography using its corresponding target [17]. They displayed an $\mathrm{scFv}$ against hen egg white lysozyme fused with filamentous phage protein III and showed that the $\mathrm{scFv}$ phages had the same pattern of reactivity as that of the parental antibody and bound to hen egg white lysozyme, with no crossreactivity with turkey egg white lysozyme, human lysozyme, or bovine serum albumin. Winter and co-workers anticipated that phage antibodies could be used in three different ways: (1) to screen small combinatorial libraries derived from antigenselected cells to rescue the original $\mathrm{V}_{\mathrm{H}}-\mathrm{V}_{\mathrm{L}}$ pairs; (2) to construct combinatorial antibody libraries to obtain entirely synthetic antibodies by combining non-rearranged $\mathrm{V}$ genes with $\mathrm{D}$ and $\mathrm{J}$ segments; and (3) to mature antibodies in vitro by the hypermutation of antigen-binding loops and subject them to further rounds of selection and mutagenesis [17].

Undoubtedly, work by Winter and co-workers on phage display technology is not only groundbreaking, but also abundant. Winter also supervised the construction of the first library of scFv antibody fragments from immunized mice to "by-pass hybridoma technology and animal immunization and [try] to build antibodies in bacteria by mimicking features of immune selection" in the future $[18,20]$. Winter and coworkers also succeeded in identifying high-affinity antibodies $\left(10^{-8} \mathrm{M}\right)$ using a random combinatorial library of the rearranged heavy $\left(\mathrm{V}_{\mathrm{H}}\right)$ and kappa $\left(\mathrm{V}_{\mathrm{k}}\right)$ light chains from mice immune to the hapten 2-phenyloxazol-5-one (phOx). Also, in 1991, Winter and co-workers published a report on the phage display of Fab fragments [16] and showed that heterodimeric Fab fragments could be assembled on the surface of the phage by linking one chain to the phage coat protein and secreting the other into the bacterial periplasm. In parallel, other groups also reported the display of Fab combinatorial libraries on the lambda phage [21]. However, this approach was abandoned for the more efficient M13 phage.

Since then, other antibody phage display systems and more refined libraries have been developed elsewhere, stimulating a competitive research environment and leading to a transformation in biotechnology.

Winter and co-workers also envisioned the humanization of antibodies [22], in which an invariant human sequence replaced the most antigenic regions of the mouse monoclonal antibody of interest. As an achievement for this technology, Winter was involved in founding Cambridge Antibody Technology, which succeeded in the humanization of the murine antitumor necrosis factor antibody suitable for chronic autoimmune disease. This antibody was approved in 2002 for the treatment of rheumatoid arthritis and became the world's best-selling drug in 2017.

\section{Outlook}

Innovations beyond the original concept of phage display have turned it into a powerful tool. Phage display technology has greatly evolved from the initial reports (Fig. 2). Since then, large numbers of phage display peptide and antibody libraries containing $10^{11-12}$ unique members have been constructed [23]. Their subsequent screening has been used to isolate novel and natural ligands for various targets and to study protein-protein interactions. The selected ligands have been used to validate the target and elucidate its structure and function.

The technology has contributed to the fields of immunotherapy and vaccine development. Several approved antibody drugs and an increasing proportion of therapeutic antibodies entering clinical trials are of human origin derived from large phage display libraries or humanization through phage display. Indeed, most known applications of phage display are related to the development of therapeutic antibodies, because they have yielded a variety of 


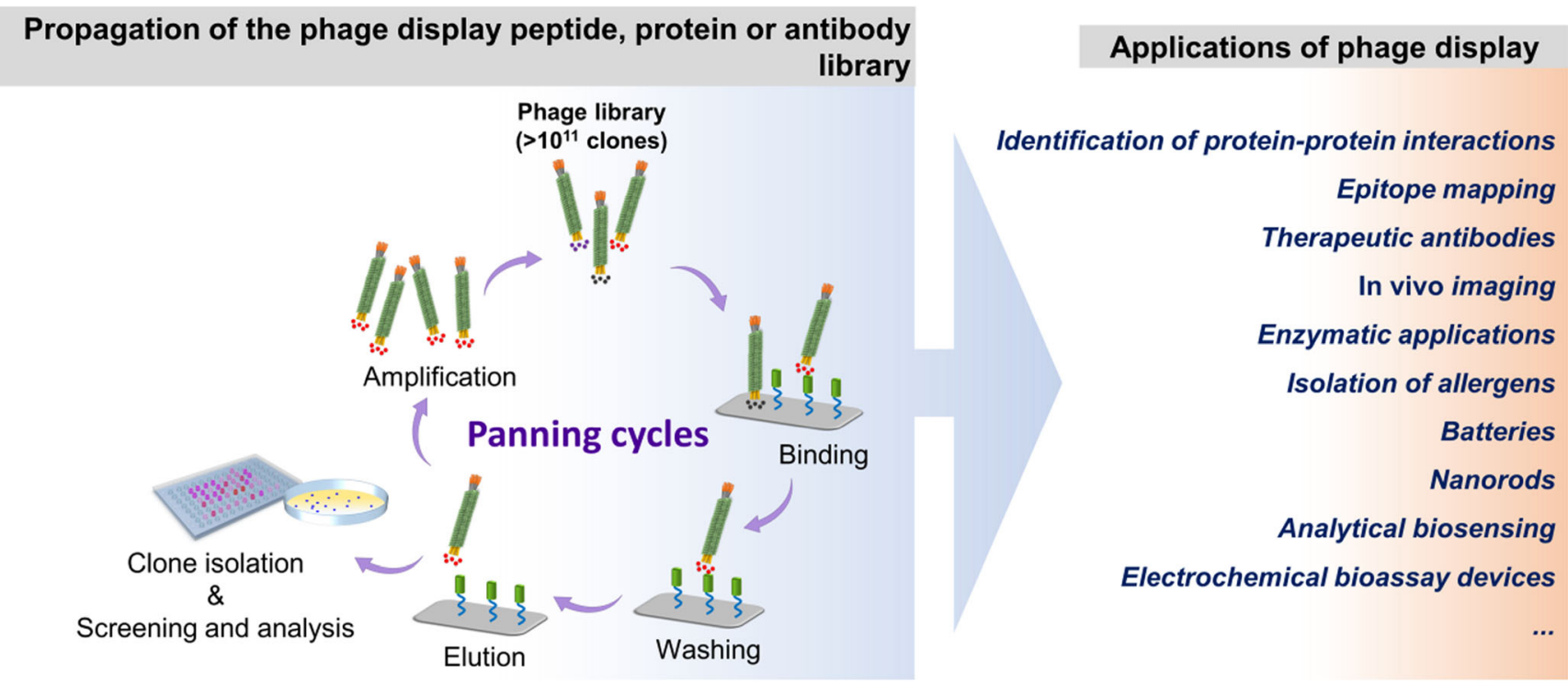

a Target presentation methods

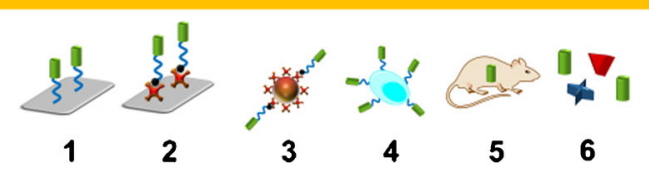

Fig. 2 Top: General scheme of the phage display biopanning procedure and applications of phage display. Bottom: (a) Description of different antigen presentation methods used to perform the biopanning procedure: (1) direct immobilization (microplates, sensor chips, immunopins, etc.); (2) immobilization through interaction with biotin-streptavidin (or neutravidin); (3) in solution with magnetic streptavidin beads; (4)

economical, accurate, and efficient biopharmaceuticals. Therapeutic antibodies obtained through this technology represent about $40 \%$ of the 20 top-selling biotechnology drugs. In addition, more than 40 approved drugs for human therapy are antibodies, and 570 are in clinical development worldwide for a wide range of diseases, including cancer, autoimmunity, inflammation, ophthalmological diseases, or organ transplantation [24, 25], with most of them derived from phage display. Importantly, phage display has produced not only massive improvements in healthcare but also vast commercial rewards.

On the other hand, the flexibility of phage display combined with the unique properties of phages, such as conservation from phage to phage, stability, or packaging, has produced a shift in engineered phages obtained through phage display from these uses to a variety of chemical and biological sensors and assays $[5,6]$. This evolution is the result of their versatility through simple manipulation of their genome, allowing easy expression on the surface of desired peptides and proteins specifically targeting high-affinity carbohydrates, proteins, or small molecules with varying functions (i.e., specific biorecognition reporters or detection protein probes), and is continuously implemented through discoveries and innovations in molecular and chemical engineering. Indeed, recent b Elution methods

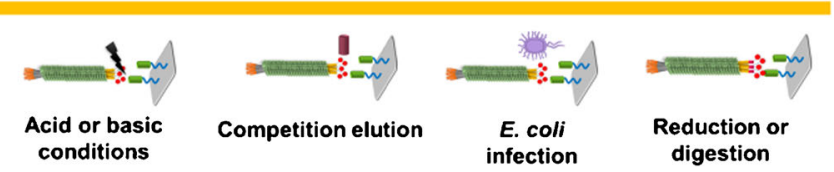

antigen-presenting cells (tumor or transfected cells); (5) in vivo screening; (6) enriched unpurified antigen or subcellular fractions. (b) The depiction of the different methods used for phage elution prior to bacterial infection. Propagation of phages is performed on bacteria, where each eluted phage clone is amplified between 10 and 100 times in each round of biopanning

advances in analytical biosensing have made use of engineered phages for their integration into different transduction methods for analytical biosensing through phage-based optical, enzymatic, colorimetric, piezoelectric mass, magnetoelastic, surface plasmon resonance, surfaceenhanced Raman spectroscopy, or electrochemical bioassay devices (for a review, see [5]). New applications of phagebased biosensing platforms will depend on the creation of new recognition elements, implementation of signal amplification strategies, or development of nanostructures for accurate geometric control.

In summary, phage display has greatly evolved in the last 25 years since the seminal report describing the technology, and its initial expected applications have become multiple (bio) applications with huge revenues. Its overall trajectory and the versatility of phage display are an indicator that exciting applications and new biopharmaceutical are on the horizon. However, the most exciting aspect in phage displaybased advances is its continuous evolution. Multifaceted selection continues to increase our ability to build massive libraries and explore their use in new research areas for phage display. Therefore, we can expect a whole new generation of products arising from the pioneering work of these 2018 Nobel laureates. 
Funding information R.B. and E. B-P. acknowledge financial support from the PI17CIII/00045 grant from the AES-ISCIII program and the CTQ2015-69278-C2-1-R/AIE grant from the Ministry of Economy and Competitiveness (MINECO), respectively.

Compliance with ethical standards The authors declare that there are no conflicts of interest regarding this manuscript.

\section{References}

1. Mann NH. The third age of phage. PLoS Biol. 2005;3:e182.

2. Matsuzaki S, Uchiyama J, Takemura-Uchiyama I, Daibata M. Perspective: the age of the phage. Nature. 2014;509:S9.

3. Lee YJ, Yi H, Kim WJ, Kang K, Yun DS, Strano MS, et al. Fabricating genetically engineered high-power lithium-ion batteries using multiple virus genes. Science. 2009;324:1051-5.

4. Zhou JC, Soto CM, Chen MS, Bruckman MA, Moore MH, Barry $\mathrm{E}$, et al. Biotemplating rod-like viruses for the synthesis of copper nanorods and nanowires. J Nanobiotechnol. 2012;10:18.

5. Peltomaa R, Lopez-Perolio I, Benito-Pena E, Barderas R, MorenoBondi MC. Application of bacteriophages in sensor development. Anal Bioanal Chem. 2016;408:1805-28.

6. Petrenko VA. Landscape phage as a molecular recognition interface for detection devices. Microelectron J. 2008;39:202-7.

7. Pasqualini R, Arap W. Hybridoma-free generation of monoclonal antibodies. Proc Natl Acad Sci U S A. 2004;101:257-9.

8. Smith GP, Petrenko VA. Phage display. Chem Rev. 1997;97:391-410.

9. Barbas CFI, Burton DR, Scott JK, Silverman GJ. Phage display: a laboratory manual. Cold Spring Harbor Laboratory Press; 2001. p. $1-736$.

10. Lee JW, Song J, Hwang MP, Lee KH. Nanoscale bacteriophage biosensors beyond phage display. Int J Nanomedicine. 2013;8: 3917-25.

11. Smith GP. Filamentous fusion phage: novel expression vectors that display cloned antigens on the virion surface. Science. 1985;228: 1315-7.

12. Parmley SF, Smith GP. Antibody-selectable filamentous fd phage vectors: affinity purification of target genes. Gene. 1988;73:305-18.

13. Scott JK, Smith GP. Searching for peptide ligands with an epitope library. Science. 1990;249:386-90.

14. Devlin JJ, Panganiban LC, Devlin PE. Random peptide libraries: a source of specific protein binding molecules. Science. 1990;249:404-6.

15. Winter G, Griffiths AD, Hawkins RE, Hoogenboom HR. Making antibodies by phage display technology. Annu Rev Immunol. 1994;12:433-55.

16. Hoogenboom HR, Griffiths AD, Johnson KS, Chiswell DJ, Hudson P, Winter G. Multi-subunit proteins on the surface of filamentous phage: methodologies for displaying antibody (Fab) heavy and light chains. Nucleic Acids Res. 1991;19:4133-7.

17. McCafferty J, Griffiths AD, Winter G, Chiswell DJ. Phage antibodies: filamentous phage displaying antibody variable domains. Nature. 1990;348:552-4.

18. Clackson T, Hoogenboom HR, Griffiths AD, Winter G. Making antibody fragments using phage display libraries. Nature. 1991;352:624-8.

19. Riechmann L, Clark M, Waldmann H, Winter G. Reshaping human antibodies for therapy. Nature. 1988;332:323-7.

20. Marks JD, Hoogenboom HR, Bonnert TP, McCafferty J, Griffiths $\mathrm{AD}$, Winter G. By-passing immunization. Human antibodies from V-gene libraries displayed on phage. J Mol Biol. 1991;222:581-97.

21. Huse WD, Sastry L, Iverson SA, Kang AS, Alting-Mees M, Burton $\mathrm{DR}$, et al. Generation of a large combinatorial library of the immunoglobulin repertoire in phage lambda. Science. 1989;246:1275-81.
22. Verhoeyen M, Milstein C, Winter G. Reshaping human antibodies: grafting an antilysozyme activity. Science. 1988;239:1534-6.

23. Hoogenboom HR. Overview of antibody phage-display technology and its applications. Methods Mol Biol. 2002;178:1-37.

24. Kaplon H, Reichert JM. Antibodies to watch in 2019. MAbs. 2018.

25. Weiner LM, Surana R, Wang S. Monoclonal antibodies: versatile platforms for cancer immunotherapy. Nat Rev Immunol. 2010;10:317-27.

Publisher's note Springer Nature remains neutral with regard to jurisdictional claims in published maps and institutional affiliations.

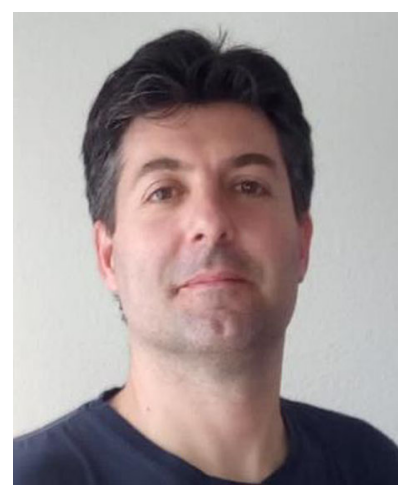

Rodrigo Barderas received his $\mathrm{PhD}$ in Chemistry (Biochemistry and Molecular Biology) from the Complutense University of Madrid (Spain) in 2004. Since 2004, he has been working in the field of proteomics and highthroughput screening techniques. In 2017, he got a Tenured Scientist position at the Instituto de Salud Carlos III. He is currently the Head of the Functional Proteomics Unit of the Chronic Disease Programme. His areas of interest include the identification of diagnostic and prognostic markers, and new targets of intervention in chronic diseases of high prevalence by using proteomics and phage display, with a special focus on colorectal cancer. He has authored over 80 research papers and book chapters.

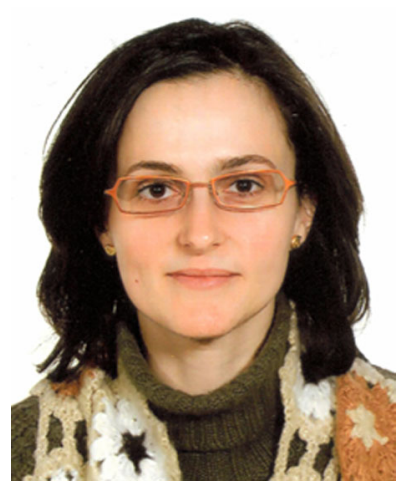

Elena Benito-Peña received her PhD in Chemistry from Complutense University (Madrid) in 2006. In 2005, she moved to the private sector working at the Chromatography Lab of the Interlab Group (now Labaqua). In 2006, she returned to Complutense University where she is currently a Postdoctoral Research Associate in the Department of Analytical Chemistry. In 2009, she was awarded with a Postdoctoral fellowship from the Spanish Government (MEC-FECYT) to work with Prof. David Walt at Tufts University (2009-2011) for the development of multiplexed optical fiber bundle-based biosensor microarrays and InfoBiology. She currently holds the position of Associate Professor at the Department of Analytical Chemistry, Complutense University of Madrid, Spain. Her research interests lie in the fabrication of micro/ nanostructured polymers and biomaterials for selective molecular recognition together with the implementation of new amplification strategies, highperformance optosensing systems, and their applications. 